\title{
Prediction of vibration and thermal behaviour of an automotive exhaust manifold: a comparative study
}

\author{
D. Desai \\ Tshwane University of Technology, South Africa
}

\begin{abstract}
Engine exhaust manifolds are commonly subjected to severe thermo-mechanical stresses caused by thermal cycles and vibrations from the engine and road profiles. The manifold is exposed to high temperature exhaust gases and is only cooled through conduction to the cylinders via thermal contact, radiation and natural convection to its surroundings. If the prevailing thermal gradients and vibrations produced by the applied loads are not properly absorbed, they can cause excessive damage and noise to the exhaust system. Hence, the prediction of the manifold's thermo-mechanical behaviour is fundamental in assessing its performance. However, modelling such behaviour is difficult as it involves complicated heat transfer processes. This paper attempts to model the transient thermal behaviour and natural frequencies of two manifolds comprising different materials in the absence of computational fluid dynamics software. The results indicate that the use of $3 \mathrm{D}$ modelling is feasible and that the stainless steel manifold potentially minimises heat loss from the exhaust gas and possess higher natural frequencies in the lower frequency domain when compared to its cast iron counterpart.
\end{abstract}

Keywords: natural frequencies, automotive exhaust manifold, transient heat transfer modelling, finite element analysis.

\section{Introduction}

In the continuous race to deliver new and innovative products faster to the market, manufacturers face many challenges such as cost reduction, industry standards, legislation, customer demands, mass reduction, shorter development cycles and satisfying customer demands. Since the first emissions legislation 
that was passed in the 1970s, automotive manufacturers have tirelessly strived to develop technologies to detoxify the exhaust. Among such technologies developed, the catalytic converter; which is installed in the exhaust system below the exhaust manifold has evolved to be most common. In order to meet the current Euro 4 emission standards, the light-off time of the catalytic converter should be less than 40 seconds (s) after a cold start [1]. This is perceived as difficult to achieve particularly for hydrocarbons (HC). Thermal inertia and heat loss through the exhaust manifold are main factors that could significantly influence the inlet gas temperature of the catalytic converter. Therefore, there are ongoing research efforts directed towards optimising heat management and minimising the time taken to reach the appropriate catalyst operating temperature.

One of the major components impacted is the exhaust manifold. Manifold design must consider various imperatives and constraints which generally lead to a compromise among contradictory requirements. Amongst them are high durability standards, low emissions, minimised vibration and heat dissipation during cold-starting, maximised heat dissipation in high temperature conditions to minimise catalyst ageing and minimising mass in order to improve fuel economy. Thermo-mechanical stresses in the system are caused by thermal cycles or thermal shocks generally caused by sudden temperature gradients in the exhaust gases produced during acceleration or deceleration of a vehicle. The catalytic converter will not be able to function effectively until it reaches its operating temperature or light-off which is in the regime of 250 to $340^{\circ} \mathrm{C}$ as reported by Burch et al. [2]. Hence, rapid warming is desirable which requires minimum heat loss in the exhaust manifold. However, at high temperatures the manifold and its related components may experience degradation by corrosion as they approach $1000^{\circ} \mathrm{C}$ as cited by Martinez et al. [3].

In order to gain a competitive advantage in the market and to study the complex thermal and mechanical phenomena inherent to exhaust manifolds, engineers have started to leverage the robust capabilities of realistic simulation to evaluate different materials for optimum performance. Also, because the time to market is constantly decreasing while the pressure to save costs is increasing, engineers seek to define and optimize the thermo-mechanical behaviour as early as possible. Examples of such studies have been conducted by Delprete et al. [4] and Deger et al. [5]. However, the phenomenon of thermo-mechanical behaviour of an exhaust manifold is complex as it comprises the simultaneous interaction of many competing factors. Some of the complexities, amongst others, are introduced by the exhaust gas flow dynamics and its interaction with the manifold itself. It is also critical to understand the different heat transfer modes involved in and around the manifold in order to investigate and improve its thermo-mechanical performance. Computational fluid dynamics (CFD) is an extremely powerful tool for such numerical investigations; however its applicability to transient modelling is limited by high computer processing demands.

Also, the mechanical aspects that influence the manifold performance are related to inputs such as vibration generated by the engine and road profiles. If 
these resonant frequencies are not treated or absorbed, they can yield excessive noise or damage. Such issues cannot be resolved by simply using finer finite element meshes, but require the development of specific models that incorporate design parameters. However, such behaviour can be predicted using computerbased models employing the finite element method. The development of such predictive models is therefore an effective and sensible approach as the flexibility of such a model can provide a powerful tool for in depth investigations without the problems often correlated with trial and error methods. The development of such predictive models therefore necessitates an intensive study of the manifold system together with the interaction of the exhaust gas.

To minimise time spent on physical experiments and improvement studies for improved catalyst light-off, many numerical investigations have been conducted augmented with experiments. Some numerical studies focused on predicting the conversion behaviours of the catalytic converters and their design optimisation, while others studied the prediction of the thermal response of the exhaust gas in the air-gap dual pipe exhaust system to improve exhaust pipe design for thermal energy preservation [6]. Yasgashi et al. [7], applied a simulation technique to optimise the heating pattern of an electrically heated catalyst while Baba et al. [8] employed a two-dimensional (2D) simulation model to investigate the effects of loading quantities of the noble metals for improved catalyst conversion efficiency. These studies have separately studied thermal response of the exhaust gas in the manifold with constant inlet gas parameters. However, none of the above models consider the engine cold-start conditions on catalyst lightoff where the wall temperatures of the manifold are gradually heated up by the exhaust gas. Such studies have only begun to appear rather recently [1] such as that investigated by Desai [9].

In this work, the finite element code ABAQUS has been employed to investigate the thermo-mechanical behaviour of a typical automotive exhaust manifold in the absence of CFD software using an alternative computational method. Constitutive models are employed to ascertain the dynamic response of these systems. Consequently, numerical models were developed and implemented in a finite element program to simulate the thermo-mechanical behaviour of the system under investigation. Within this framework, a stainless steel manifold is compared to a typical cast iron manifold of exactly the same configuration (geometry and dimensions). Both cases were produced by the casting process. This numerical comparison may provide some indication in terms of the natural vibration characteristics and catalyst light-off time although the objective is not to quantify the difference in terms of actual time taken to reach light-off, but rather to qualitatively indicate the potential effect of the thermal properties of different manifold materials.

The insights gained into the thermo-mechanical behaviour of the manifold under investigation may materialize into realistic design modifications and can augment existing studies enabling engineers to move closer towards exhaust manifold refinement in pursuit of their quest for optimal designs. 


\section{Mathematical models}

Heat will be transferred from the exhaust gas to the manifold by forced convection and radiation, and dissipated via the following processes:

a) A portion of the heat transferred by the gas will be stored in the material, the importance of which will depend on the thermal mass (mass $\times$ specific heat). This amount of heat energy will be close to zero once the exhaust system has reached thermal equilibrium (fully warmed up).

b) The remaining portion of the heat transferred by the gas will be transmitted to the environment by radiation and possibly by free or forced convection, depending on the exhaust system's architecture. This portion of heat energy is initially very small but becomes more significant once the system reaches high temperatures.

Since the actual flow in the manifold is unsteady and compressible, the flow condition at any point of interest may be described by three independent variables, i.e. pressure, velocity and density. However, since the focus of this paper is on the thermal effects on the exhaust system on a comparative basis, the effect of flow pulsation due to pressure wave actions acting on the heat transfer process is neglected. By assuming a quasi-steady, incompressible flow, the one dimensional (1D) energy equation for the exhaust gas and manifold wall may be represented as

$$
\frac{\partial T_{\mathrm{m}}}{\partial t}=\alpha \frac{\partial^{2} T_{\mathrm{m}}}{\partial x^{2}}+\frac{q_{\mathrm{gm}}-q_{\mathrm{eva}}-q_{\mathrm{rad}}}{\rho_{\mathrm{m}} c_{\mathrm{p}} V_{2}}
$$

where $T_{\mathrm{m}}$ is the temperature of the manifold, $q_{\mathrm{gm}}$ is the heat flux for convection between the exhaust gas and manifold wall and $q_{\text {eva }}$ and $q_{\text {rad }}$ represents the evaporative and radiative flux, respectively. $c_{\mathrm{p}}$ is the heat capacity of the manifold, $\rho_{\mathrm{m}}$ the density of the manifold, $V_{2}$ is the volume of the manifold segment. Eqn. (1) is actually the unsteady heat conduction equation with a uniform thermal conductivity applied to the manifold wall. The spatial and temporal temperature distribution $T(x, y, z, t)$ for 3D heat conduction in a domain $\mathrm{D}$ is given as:

$$
\frac{\partial}{\partial x}\left(\kappa_{x} \frac{\partial T}{\partial x}\right)+\frac{\partial}{\partial y}\left(\kappa_{y} \frac{\partial T}{\partial y}\right)+\frac{\partial}{\partial z}\left(\kappa_{z} \frac{\partial T}{\partial z}\right)+Q=\rho c\left(\frac{\partial T}{\partial t}-V \frac{\partial T}{\partial x}\right)
$$

where $Q$ is the power generation per unit volume, $k$ the thermal conductivity and $(x, y, z)$ the coordinate system.

Heat loss due to thermal radiation between the manifold surface and environment is modeled by the standard Stefan-Boltzmann relation

$$
q_{\mathrm{rad}}=\varepsilon \sigma\left(T_{\mathrm{m}}^{4}-T_{\mathrm{a}}^{4}\right)
$$

where $\varepsilon$ is the emissivity, $T_{\mathrm{a}}$ is the air temperature and $\sigma$ is the standard Stefan-Boltzmann constant. 
Heat convection from exhaust gas to manifold wall is mainly due to forced convection and is strongly dependent on the gas flow dynamics and the manifold geometry. Neglecting the water film layer on the inner wall of the manifold due do quenching of the hot exhaust gas during engine cold-start, the heat flux may be expressed as

$$
q=h_{\mathrm{gm}}\left(T_{\mathrm{g}}-T_{\mathrm{m}}\right)
$$

where $T_{\mathrm{g}}$ is the temperature of the exhaust gas and $h_{\mathrm{gm}}$ is the convective heat transfer coefficient between the exhaust gas and the manifold wall, and is determined by

$$
h_{\mathrm{gm}}=N_{u} \frac{k}{d_{1}}
$$

where $k$ is the thermal conductivity of the gas, $N_{\mathrm{u}}$ the Nusselt number and $d_{1}$ the manifold inner diameter. The widely used Nusselt correlation, neglecting manifold bend and gas pulsation factors, cited by Hussein et al. [10] and Mills [11] was employed.

$$
\text { For } 0.5<\operatorname{Pr}<2000 \text { and } 10^{4}<\operatorname{Re}_{\mathrm{D}}<5 \times 10^{6}
$$

Heat convection from manifold surface to ambient air maybe modelled as a heat flux, which from the manifold outer surface to the ambient air $q_{\text {cva }}$ due to free convection can be represented by

$$
q_{\mathrm{cva}}=h_{\mathrm{cva}}\left(T_{\mathrm{m}}-T_{\mathrm{a}}\right)
$$

where

$$
h_{\mathrm{cva}}=\frac{N_{u} k_{\mathrm{a}}}{d_{2}}
$$

The Nusselt number is determined from the correlation proposed by Churchill and Chu [12] as cited by Bergman et al. [13] and is represented as

$$
N_{u}=0.6+\left(\frac{0.387 R a^{1 / 6}}{\left[1+(0.599 / P r)^{9 / 16}\right]^{8 / 27}}\right)
$$

where

$$
R a=\frac{l^{3} g \beta\left(T_{\mathrm{p}}-T_{\mathrm{a}}\right)}{v_{\mathrm{a}}^{2}} P r
$$

and the wetted length

$$
l=\pi d_{2}
$$


The volumetric thermal expansion coefficient $\beta$ is evaluated at $T_{\mathrm{a}}$ while the other properties are evaluated at the film temperature $T_{\mathrm{f}}$ which may be calculated from

$$
T_{\mathrm{f}}=T_{\mathrm{p}}-0.38\left(T_{\mathrm{p}}-T_{\mathrm{a}}\right)
$$

For the calculation of the manifold's coupled natural frequencies neglecting damping and reactivity, the basic eigenvalue problem can be stated as

$$
\left[\begin{array}{cc}
K^{N M} & -A^{T} \\
0 & K^{P Q}
\end{array}\right]\left\{\begin{array}{l}
u^{M} \\
p^{Q}
\end{array}\right\}-\omega^{2}\left[\begin{array}{cc}
M^{N M} & 0 \\
A & M^{P Q}
\end{array}\right]\left\{\begin{array}{l}
u^{M} \\
p^{Q}
\end{array}\right\}=0
$$

where $\omega^{2}$ is the eigenvalue, the superscripts $P, Q$ refer to pressure degrees of freedom in the fluid, A matrix representing coupling between the structure and the fluid and $N, M$ refers to the displacement degrees of freedom in the manifold. All eigenvectors in this study are normalised with respect to displacement, U.

\section{Thermo-mechanical modelling}

\subsection{Description and geometry}

In this work, a 3D finite element model for simulating the natural frequencies and transient heat transfer process in the exhaust manifold of a typical sedan vehicle during a cold-start is developed employing the ABAQUS finite element code. Appropriate convective and radiative boundary conditions are considered. The problem domain comprises an exhaust manifold subjected to isothermal exhaust gas flow. A CAD model of the physical manifold depicted in fig. 1, suitable for finite element analysis was developed from surface data with the actual geometry and complexity of the physical manifold retained.
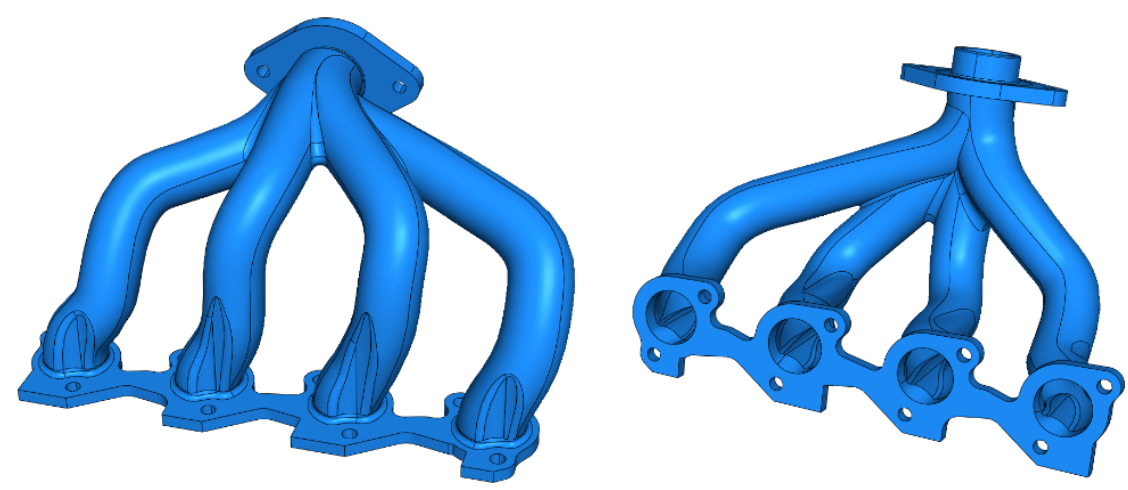

Figure 1: Solid model of exhaust manifold. 
The modelling sequence based on sections 3.3 and 3.3 below, was according to the following steps:

1) A cold modal analysis was run to determine the eigenfrequencies.

2) A thermal analysis was then run to predict the temperature distribution.

3) Finally, a hot modal analysis was done to determine the eigenfrequencies with the imposed thermal load captured in step 2 above.

\subsection{Material model}

Non-linear material models for both stainless steel (310) and cast iron (SiMo) are used as their thermo-mechanical response varies over its temperature range. Transient heat transfer analysis also requires the specification of thermal conductivity, specific heat, film coefficients and material density.

\subsection{Loading and interaction conditions}

The initial temperature was set to $300 \mathrm{~K}$ in the entire model. The manifold exterior is exposed to air that has a constant temperature of $300 \mathrm{~K}$. A total mass flow rate of $0.0808 \mathrm{~kg} / \mathrm{s}$ with gas inlet and outlet temperatures of $823 \mathrm{~K}$ and $623 \mathrm{~K}$ were used. No temperature boundary conditions were applied. The thermal response of the model is driven entirely by thermal loading through film coefficients.

The inner manifold surfaces are in contact with the exhaust gas. Heat flux on the inner manifold surfaces is applied by a film condition. Using the equations described in section 2, the heat transfer coefficient, (neglecting entrance, manifold bend and pulsation factors) was analytically computed as approximately $270 \mathrm{~W} / \mathrm{m}^{2} / \mathrm{K}$, similar to that calculated by Zidat and Parmentier [14]. The outer surfaces, initially associated with a sink temperature of $300 \mathrm{~K}$, are exposed to free air, which has a film coefficient of $15 \mathrm{~W} / \mathrm{m}^{2} / \mathrm{K}$. Emissivities of 0.2 and 0.8 were prescribed for stainless steel and cast iron, respectively.

\subsection{Finite element mesh}

Both second-order tetrahedral diffusive heat transfer elements and continuum structural elements were used to discretise the manifold. Due to the complexity of the model, a global mesh seed of approximately $2 \mathrm{~mm}$ was assigned on the prevailing unstructured mesh. The model size was approximately 710000 degrees of freedom.

\section{Results and discussion}

Figs. 2 and 3 show the temperature distribution of the exhaust manifolds at $40 \mathrm{~s}$ in kelvin. The simulation of the steel and cast iron models converged at 23.38 hrs and 28.52 hrs of CPU time, respectively.

The simulations reveal that both materials exhibit similar temperature profiles under the operating conditions described. Although cast iron has a higher 


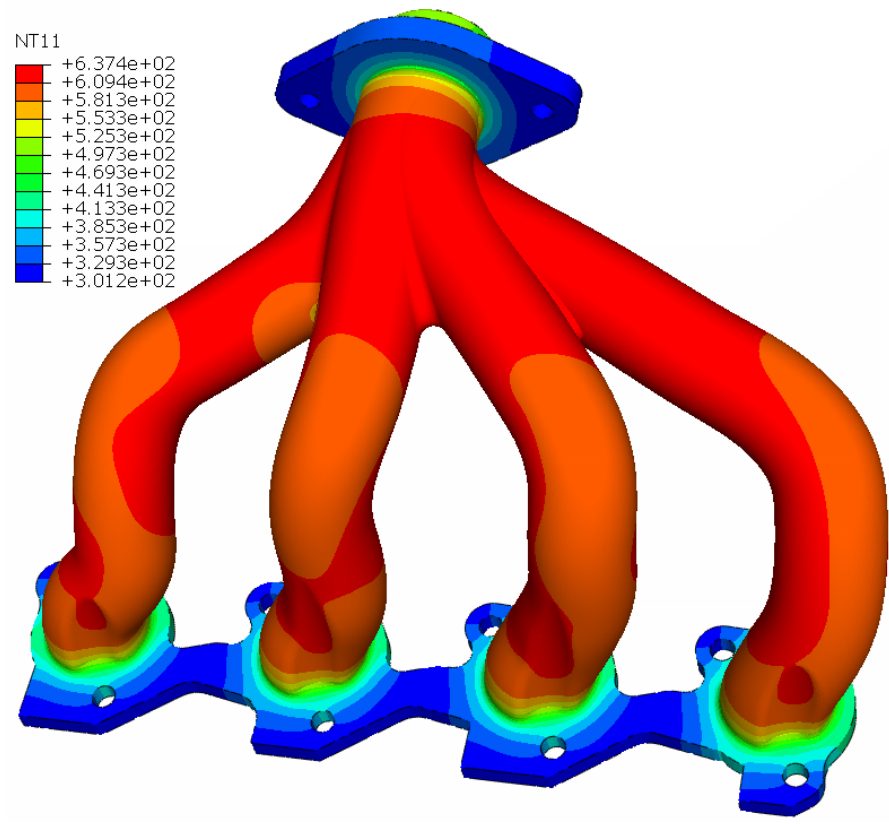

Figure 2: Temperature profile in the steel manifold at $40 \mathrm{~s}$.

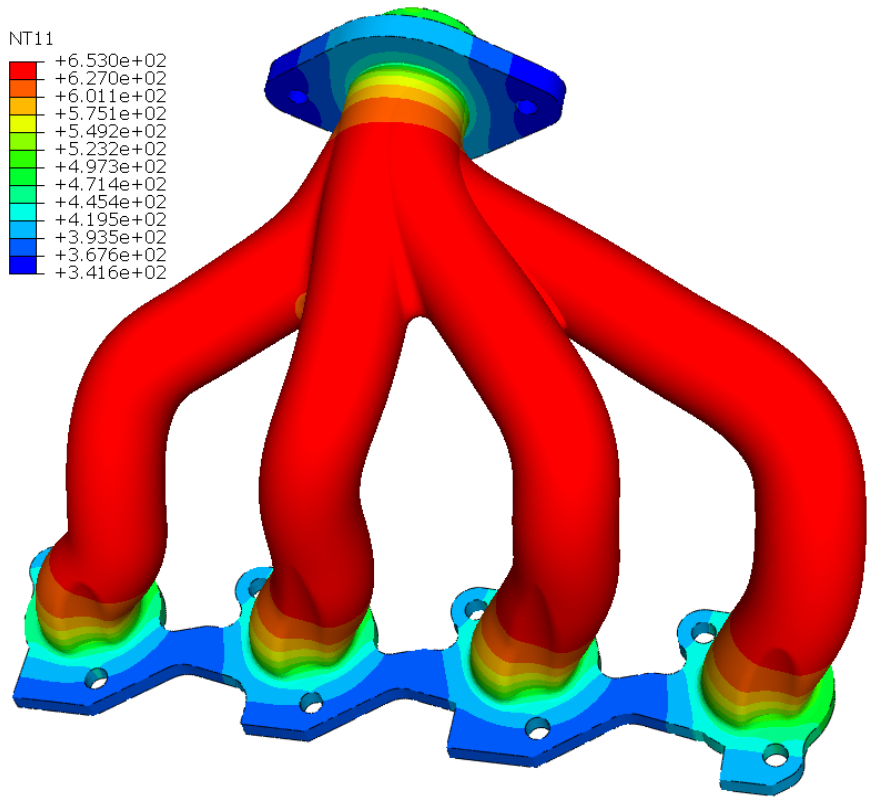

Figure 3: Temperature profile in the cast iron manifold at $40 \mathrm{~s}$. 
thermal conductivity than steel, the results, based on the contour plots indicate that the cast iron manifold has only a slightly higher overall outer wall temperature than the steel manifold. This may be due to the significant difference in emissivities between the two materials and to the heat transfer modes of conduction and surface radiation competing in a complicated way. This implies that contrary to popular opinion, a material having a higher thermal conductivity does not necessarily dominate the surface temperature distribution. It can be further realised that the surface temperature distribution depends on many competing factors with the internal heat transfer coefficient being the most dominant mode of heat loss from the manifold.

Also, as expected in both cases of the hot modal analyses, the eigenvalues are lower since most materials yield with increasing temperature. This is evident in table 1 below. Furthermore, the decrease in eigenfrequencies is much lower in the stainless steel manifold than its cast iron counterpart as the material response of cast iron varies drastically over its temperature range.

Table 1: $\quad$ Eigenfrequencies of cold and hot models (Hz).

\begin{tabular}{|l|c|c|r|r|c|}
\hline Model/Mode & Mode 1 & Mode 2 & Mode 3 & Mode 4 & Mode 5 \\
\hline Cold steel & 804.21 & 952.10 & 988.74 & 1312.00 & 1407.40 \\
\hline Hot steel & 803.80 & 951.62 & 988.23 & 1311.30 & 1406.70 \\
\hline Cold cast iron & 372.95 & 825.90 & 1114.00 & 1334.30 & 1911.30 \\
\hline Hot cast iron & 346.53 & 770.79 & 1039.90 & 1250.20 & 1779.80 \\
\hline
\end{tabular}

The comparison of mode shapes between the hot and cold models exhibits similar mode shapes in both cases. Fig. 4 shows as an example the first mode shapes of the hot and cold steel manifold.

\section{Conclusion}

This paper proposes an alternative procedure to CFD for predicting the thermomechanical behaviour of two exhaust manifolds comprising different materials. This alternate procedure was chosen since the applicability of CFD is usually limited to steady-state simulations due to high computational times required for transient simulations. This work has presented the thermal distribution due to the flow of hot exhaust gas as well as results from modal analyses under hot and cold conditions. The results indicate that the use of 3D modelling is feasible and that the stainless steel manifold minimises heat loss from the exhaust gas and possess higher natural frequencies in the low frequency range compared to its cast iron counterpart. However, at higher frequencies, the steel manifold exhibits lower natural frequencies than that of the cast iron manifold. It can also be seen that the transient thermal performance of an exhaust manifold is governed by complex interactions between the exhaust gas flow and the manifold itself. It is hoped that this research will make deeper inroads into how exhaust manifolds behave under complex loading conditions and would be of benefit in future designs in the quest for quieter and more durable manifolds. 


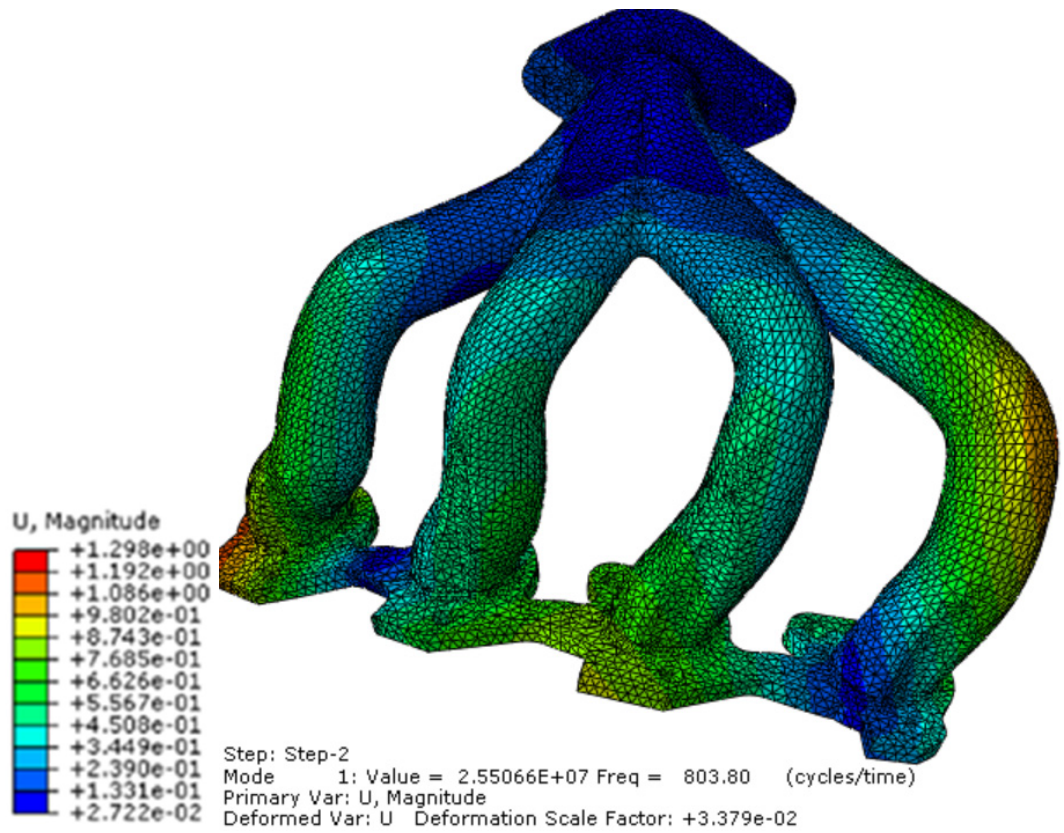

(a)

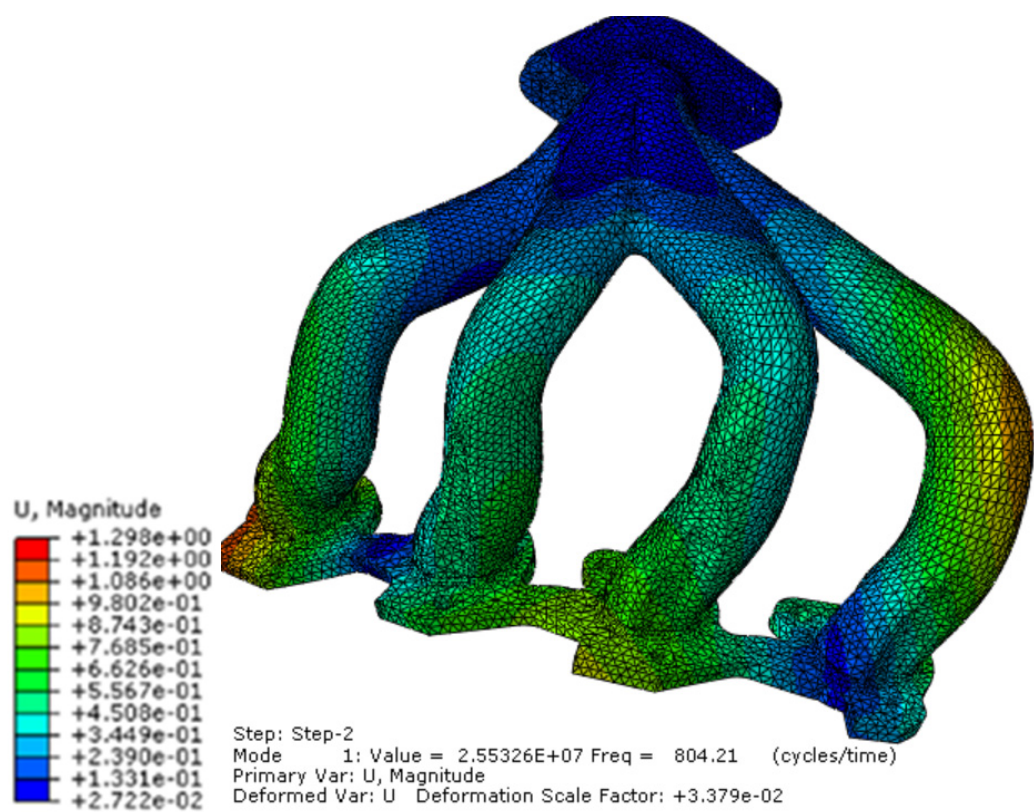

(b)

Figure 4: Fundamental mode shape of steel manifold (a) hot; (b) cold. 


\section{References}

[1] Chan, S.H. \& Hoang, D.L., Heat transfer and chemical reactions in exhaust system of a cold-start engine, International journal of heat and mass transfer, 42, 4165-4183, 1999.

[2] Burch, S.D., Porter, T.F., Keyser, M.A., Brady, M.J \& Michaels, K.F., Reducing cold start emissions by catalytic converter thermal management, Society of Automotive Engineers, SAE paper 950409, 1995.

[3] Martinez, N., Amado, M. \& Guerrero, M.P., Modeling the vibrations in a catalytic converter for diesel engine. SIMULIA Customer Conference, Vienna, Austria, pp. 1-7, 2011.

[4] Delprete, C., Sesana, R. \& Vercelli, A., Multiaxial damage assessment and life estimation: application to an automotive exhaust. Procedia Engineering, Fatigue 2010, (2): 725-734, 2010.

[5] Deger, Y., Simperl, B. \& Jimenez, L.P., Coupled CFD-FE analysis for the exhaust manifold of a diesel engine. ABAQUS User's Conference, Milan, Italy, pp. 199-208, 2004.

[6] Koltsakis, G.C., Konstantinidis, P.A. \& Stamatelos, A.M., Development and application range of mathematical models for 3-way catalytic converters. Applied Catalysis B: Environmental, 12: 161-191, 1997.

[7] Yasgashi, T., Yoshizake, K., Nagami, T., Sugiura, S., Yoshimaga, T. \& Ohsawa, K., New technology for reducing the power consumption of electrically heated catalysts. Society of Automotive Engineers, SAE paper 940464, 1994.

[8] Baba, N., Ohsawa, K. \& Sugiura, S., Numerical approach for improving the conversion characteristics of exhaust catalysts under warming-up condition. Society of Automotive Engineers, SAE paper 962076, 1996.

[9] Desai, D.A., A comparative non-linear simulation of temperature profiles induced in an exhaust manifold during cold-starting. Journal for New Generation Sciences, 8(1): 47-63, 2010.

[10] Hussein, A.M., Bakar, R.A., Kadirgama, K. \& Sharma, K.V., Simulation study of turbulent convective heat transfer enhancement in heated tube flow using $\mathrm{TiO}_{2}$-water nanofluid. $2^{\text {nd }}$ International Conference on Mechanical Engineering Research, Materials Science and Engineering, 50: 1-6, 2013.

[11] Mills, A.F., Basic heat and mass transfer. New international ed., Prentice Hall, New Jersey, 2013.

[12] Churchill, S.W. \& Chu, H.H.S., Correlating equations for laminar and turbulent free convection from a horizontal cylinder. International journal of heat and mass transfer, 18: 1049-1053, 1975.

[13] Bergman, T.L., Lavine, A.S., Incropera, F.P. \& De Witt, D.P., Introduction to heat transfer. $6^{\text {th }}$ ed., John Wiley \& Sons, Inc., 2011.

[14] Zidat, S. \& Parmentier, M., Exhaust manifold design to minimize catalyst light-off time. Society of Automotive Engineers, SAE paper 2003010940, 2003. 